\title{
BMJ Open Development of a core outcome set to use in the research and assessment of malignant bowel obstruction: protocol for the RAMBO study
}

\author{
Elin Baddeley (D) , ${ }^{1}$ Alison Bravington, ${ }^{2}$ Miriam Johnson, ${ }^{2}$ David C Currow, ${ }^{2,3}$ \\ Fliss EM Murtagh, ${ }^{2}$ Elaine Boland, ${ }^{4}$ George Obita, ${ }^{5}$ Annmarie Nelson, ${ }^{1}$ \\ Kathy Seddon, ${ }^{1}$ Alfred Oliver, ${ }^{6,7}$ Simon Noble, ${ }^{1}$ Jason Boland (D) $^{2}$
}

To cite: Baddeley E,

Bravington A, Johnson M, et al. Development of a core outcome set to use in the research and assessment of malignant bowel obstruction: protocol for the RAMB0 study. BMJ Open 2020;10:e039154. doi:10.1136/ bmjopen-2020-039154

- Prepublication history for this paper is available online. To view these files, please visit the journal online (http://dx.doi. org/10.1136/bmjopen-2020039154).

Received 06 April 2020 Revised 22 May 2020 Accepted 29 May 2020

Check for updates

(C) Author(s) (or their employer(s)) 2020. Re-use permitted under CC BY-NC. No commercial re-use. See rights and permissions. Published by BMJ.

For numbered affiliations see end of article.

Correspondence to

Elin Baddeley;

baddeleye1@cardiff.ac.uk

\section{ABSTRACT}

Introduction Studies regarding the management of malignant bowel obstruction (MBO) report conflicting findings. This is partly due to different outcome measures being used to evaluate severity of $\mathrm{MBO}$ and the response to treatments. Furthermore, current outcome measures focus mainly on measurable physiological parameters which may not correlate strongly with patient-defined quality of life. The development of core outcome sets allows a consistent approach to evaluating clinical conditions taking into consideration patient, healthcare professional and researcher viewpoints. It follows an internationally recognised standard methodology. We present a protocol for the development of a core outcome set for Research and Assessment of MBO (RAMBO). Methods RAMBO is a multicentre study, comprising of four phases: a systematic review to examine current scope of outcome measures associated with MBO (phase I). Interviews with patients, companions and healthcare professionals will explore priorities and preferences for care and outcomes (phase II). An expert panel meeting will collate the findings into a set of outcomes (phase III), refined by consensus through a Delphi survey with key stakeholders (phase IV). The final set of outcomes will be ratified at a consensus meeting. Each step will actively include patient partners. Thematic analysis and descriptive statistics will be used to analyse qualitative and quantitative data, respectively.

Ethics and dissemination Ethical approval was obtained (Wales REC 5, REF: 19/L0/1876). Study participants and relevant stakeholders will be updated with newsletters and a lay summary at the end of the study. Abstracts will be submitted to national and international conferences, result papers will be submitted to peer-reviewed, open access journals.

Trial and PROSPERO registration numbers Core Outcome Measures in Effectiveness Trials (1402); Systematic Literature Review (CRD42019150648); Rapid Review (CRD42020176393).

\section{INTRODUCTION}

Malignant bowel obstruction (MBO) is bowel obstruction due to cancer or its treatments. It primarily affects people with advanced
Strengths and limitations of this study

- This is the first study to develop a core outcome set for the evaluation of malignant bowel obstruction.

- The protocol follows a well-established process following Core Outcome Measures in Effectiveness Trials guidelines, including a thorough systematic review, qualitative rapid review, semistructured interviews with patients, their companions and healthcare professionals, followed by a three-step Delphi survey.

- The study outcomes will reflect what is important to patients and their companion/carers as well as clinicians and researchers.

- Patient partner involvement (PPI) for this study adheres to the UK PPI standards and has occurred from the inception of the study, particularly the methodology, including data collection tools for patient and companion interviews.

- Since the qualitative data collection will occur in the UK, there may be limitations on how applicable the outcome measures will be if applied internationally.

ovarian $(10 \%-50 \%)$ and gastrointestinal cancers $(15 \%)$ and remains a challenging condition facing oncology, surgical, gynaecology, gastroenterology and palliative care teams. ${ }^{12}$ It is one of the most distressing complications of cancer, causing nausea, vomiting, abdominal distension, colic, pain and constipation. ${ }^{3-8}$ It also has a persistent and profound effect on quality of life ${ }^{9}$ and often requires hospitalisation for persistent unresolved symptoms. ${ }^{3}$

Management options for MBO in patients with advanced cancer are limited; some, usually those with a single-level bowel obstruction, may be offered surgery to resect an isolated tumour or create a defunctioning stoma. Some people may benefit from the endoscopic placement of a stent for palliative purposes. However, the majority of 
cases present with multiple level obstructions which are not amenable to surgical or endoscopic interventions. Pharmacological approaches for symptom reduction in inoperable MBO (IMBO) usually involve a combination of antiemetics and parenteral antisecretory agents (anticholinergics, somatostatin analogues and $\mathrm{H}_{2}$ antagonists), often with gut decompression (nasogastric tube or venting gastrostomy). ${ }^{1011}$

Current evidence informing the symptom management of IMBO is inadequate; clinical guidelines favour somatostatin analogues but recommendations have been based, largely, on case series and insufficiently powered clinical trials. ${ }^{9}$ Recently, two adequately powered, well-conducted clinical trials demonstrated no objective benefit, with one of them suggesting evidence of harm. ${ }^{4}{ }^{12}$ Importantly, these two trials included a placebo arm to reflect the natural history of IMBO, which is largely unknown, except that spontaneous resolution with recurrence is common and, for many people, it appears that vomiting will generally lessen in frequency and volume with time. However, these trials do not appear to have impacted on subsequent guidelines or clinical recommendations ${ }^{13-16}$; although there are some data showing they have affected clinical practice. $^{17}$

A recent systematic review of somatostatin analogues, using objective tools to evaluate data quality, concluded that there was moderate evidence of no benefit and only poor evidence for their use. ${ }^{18}$ The authors suggested that the most significant challenge in evaluating current data lays with the many studies using differing clinical outcomes, thereby making it impossible to objectively compare study outcomes or submit them to meta-analysis. The lack of an agreed core outcome set (COS) for MBO has considerable challenges in ensuring consistent clinical and research evaluation of interventions for patients with $\mathrm{MBO}$.

\section{Identifying outcome measures that reflect real-world settings}

In order to ensure that outcome measures are relevant to patients, it is essential to have patients at the frontline of the research in order to ascertain their quality of life and preferences for symptom management and care. Patient-reported outcomes, when collected and analysed appropriately, are key to shared decision-making, clinical guidelines and health policy. ${ }^{19}$ Involving patients' views and understanding the challenges they face are invaluable in understanding how care happens in real-world settings, otherwise trials might fail to account for the outcomes that matter most to patients. ${ }^{20}$

\section{Rationale of COS development for MBO}

A key issue identified on both national and international levels is the lack of data indicating how primary outcome measures used to evaluate MBO related to patient experience. ${ }^{1{ }^{17}}$ For example, measures of symptom relief may not directly reflect what is important to patients and their companions (next of kin/carers) and what they consider to be meaningful. ${ }^{18}$
The broad and varied approaches to outcome measurement in the palliation of $\mathrm{MBO}$, and the lack of an evidence base for patient-relevant measures, have led to a diversity of approach in clinical trials and inconsistent recommendations in clinical guidelines. Importantly, the continuation of poorly evidenced clinical practice may also cause harm. ${ }^{21}$ Recent publications have called for the development of a COS, to ensure uniformity of symptom assessment within clinical trials and clinical practice. ${ }^{21}$ This study aims to address this gap. It will ascertain patients' perceptions of the management of their condition, compare these with outcomes measured in the current literature and gain consensus on an agreed set of core outcomes to be used in clinical practice to streamline appropriate and targeted management of MBO.

\section{Aims}

The Research and Assessment Outcome Measures for MBO (RAMBO) study aims to review the current scope of outcome measures used to evaluate $\mathrm{MBO}$ and to develop a set of core outcomes specific to MBO. It will ask the following questions:

- What outcome measures are currently used to evaluate $\mathrm{MBO}$ within the context of clinical research and clinical practice?

- What symptoms and effects do patients with MBO, their companions and healthcare professionals consider most important, and what improvement would they consider clinically meaningful?

- How do currently used outcomes map to those factors identified as important by patients, their companions and healthcare professionals, and what are the gaps and limitations in current measures?

\section{METHODS}

\section{Study design}

We propose to use a multiphase, mixed-method, multicentre study to address the research questions. In phase I, a systematic literature review (SLR) will be conducted to identify outcomes currently used to evaluate MBO. Phase II will comprise of interviews with patients, their companions and healthcare professionals to understand their experiences, priorities and preferences for care. The findings of these two phases will inform phase III, an expert panel meeting, to direct the compilation of a comprehensive list of outcomes which will feed into the phase IV Delphi survey, a two-round ranking exercise followed by a consensus meeting. The aim of the Delphi is to achieve consensus on a final COS. A summary of the design is seen in figure 1. Dissemination will follow the COS-STAndards for Reporting Statement. ${ }^{22}$

\section{Patient and public involvement}

We actively support the fundamental involvement of patient and public involvement as set out by the UK Standards for Public Involvement in research in all of our research. ${ }^{23}$ The RAMBO study has recruited two research partners (one of who is a coapplicant on the study) to 


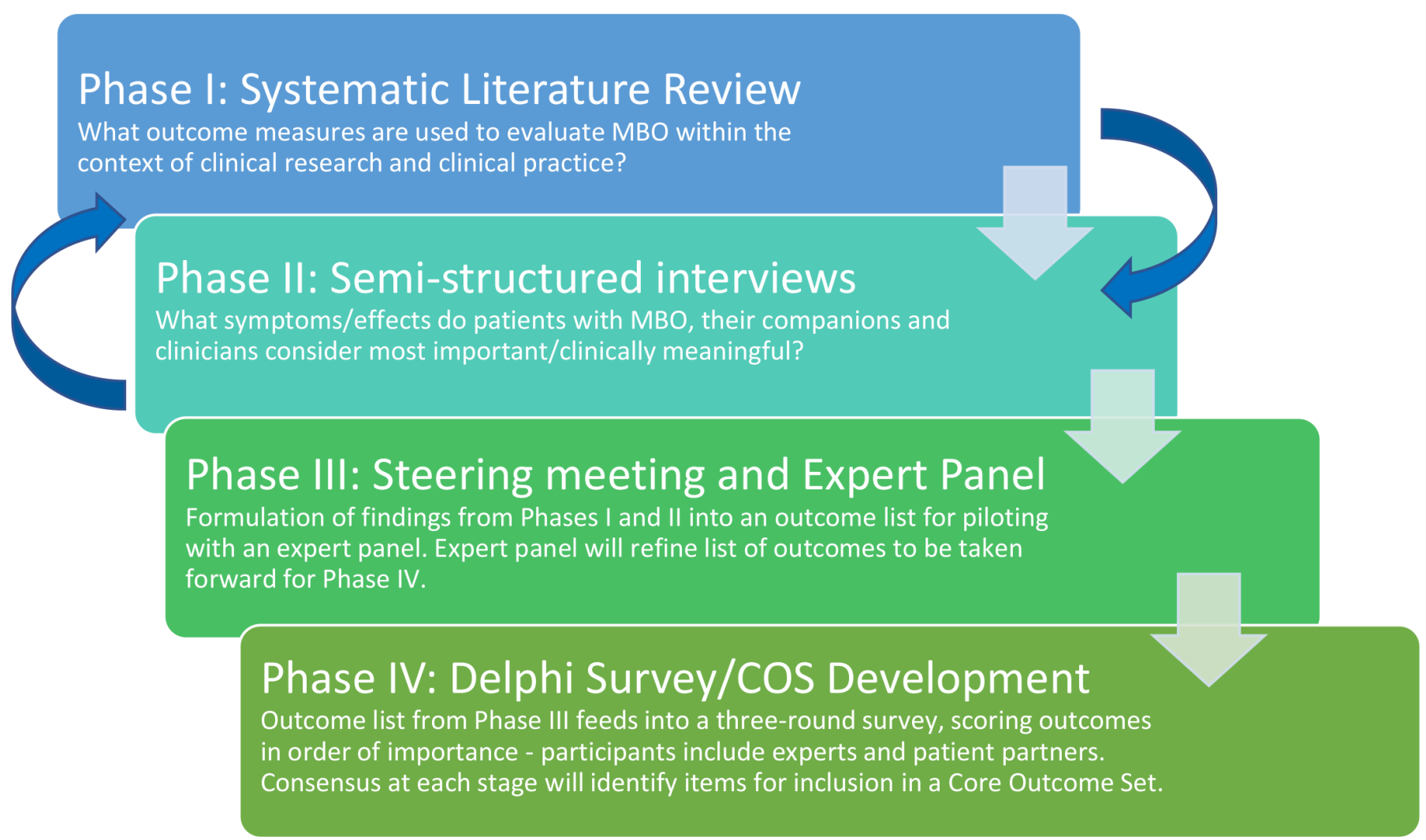

Figure 1 Process for the development of a core outcome set (COS) for malignant bowel obstruction (MBO).

represent lay stakeholders in the study management group advising on recruitment and participant-facing documentation. They will also contribute to thematic analysis of the interview data. Lay representatives will also be involved in the interpretation and dissemination of the study results.

\section{Phase I: SLR}

The Core Outcome Measures in Effectiveness Trials (COMET) Initiative recommends an SLR as an initial phase in studies developing COS. ${ }^{22}$ This will identify all outcomes used in clinical trials and observational studies in patients undergoing palliative treatment for inoperable MBO. Qualitative papers will not be included as they do not quantify outcomes, but a separate, rapid review of qualitative studies will inform the writing up of the phase II interview study (see below). Databases to be searched include MEDLINE, EMBASE, CINAHL, CareSearch and the Cochrane Central Register of Controlled Trials. Other sources will include reference lists of included articles and of previous systematic reviews of MBO interventions, conference proceedings, grey literature and the authors' own files. No date or language restrictions will be imposed.

The research questions that the review seeks to answer are:

- What outcome measures are used to evaluate clinical, symptomatic and psychological aspects of $\mathrm{MBO}$ within the context of clinical research and practice?

- How is the timing of outcome measures justified?
- Which outcome measures should be considered for inclusion in a COS for future clinical trials?

A broad range of study designs and heterogeneous data are anticipated in papers meeting the inclusion criteria for this review, and data will be explored using narrative synthesis. The review will record all clinical, symptomatic and psychological outcomes reported in the included studies, with the definitions and time points of each measure, and will review their heterogeneity and their relevance to patients with MBO. Data will be extracted and grouped with reference to the outcome taxonomy recommended by COMET. The full protocol for the review, including the search strategy and study selection criteria, is available on the PROSPERO database.

\section{Phase II: interviews with patients, their companions and healthcare professionals \\ Participant eligibility and sampling}

Interviews will explore the experiences of approximately 15 patients with MBO, what outcomes they would most value from their care and the experiences of their companion if possible. In this case, companion if defined as a significant informal carer or family member (aged over 18). Patients eligible for interview will be adults aged 18 or more with histologically confirmed cancer, with their MBO currently or recently resolved but with risk of recurrence, as confirmed by either two senior medical practitioners or one medical practitioner in combination with confirmatory radiology. Patients will be interviewed with their carer/companion present if this is the patient's 
preference; carers/companions will be interviewed separately where this is preferred. We will not include patients who have any diagnosed medical problems that would prohibit them from participating in a $30 \mathrm{~min}$ to 1 hour interview.

We will also interview approximately 15 healthcare professionals from a breadth of clinical specialities involved in the care of patients with $\mathrm{MBO}$ at the recruitment sites in the 12 months prior to recruitment, to explore the experiences involved in the management of MBO. Healthcare professionals will be purposively sampled to ensure a breadth of clinical specialties involved in the management of $\mathrm{MBO}$, including primary care, palliative care, oncology, colorectal and gynaecological surgery.

The sample size of participants is based on the past experience of patients receiving palliative care services across multiple studies and aims to reach data saturation-the point at which no new topics emerge. This will be achieved by constant reevaluation and discussion between the two interviewers and with input from the study management team.

\section{Data collection}

A semistructured interview format will be used to explore patients' understanding, experiences and symptoms of $\mathrm{MBO}$, what matters most to patients with $\mathrm{MBO}$ and what they consider to be a meaningful improvement regarding the relief of MBO symptoms. Interviews conducted with healthcare professionals will explore their experiences of managing $\mathrm{MBO}$, how they evaluate severity and symptomatic response to treatment, what they perceive matters most to patients in regard to treatment of $\mathrm{MBO}$ and how they perceive 'meaningful improvement' in a patient with MBO.

Interviews are expected to last between $30 \mathrm{~min}$ and 1 hour. They will be undertaken in a quiet location at participating hospitals or hospices or an alternative location of the participants' choice, at a time specified by the participant.

Interviews will be audio recorded on an encrypted recording device (with the participants' consent) and uploaded onto a secure server, labelled with an identifying code, with no reference to personal details. Audio files will be transcribed verbatim by an authorised agency. Basic demographic data will be collected and reported descriptively but not used as sampling criteria. Transcripts will be checked to ensure anonymisation by a member of the research team before being uploaded to NVivo V.12 ${ }^{24}$ qualitative software for data management.

\section{Consent}

Written consent will be obtained at the outset of each interview. Individuals who lack the capacity to consent will not be included in this study. Participant information sheets will be distributed to potential participants by their clinical team, to give participants the time to make an informed decision whether or not they wish to participate. If they choose to participate, they will inform a member of their clinical team, who will pass on the patients' contact details to the researcher or the patient can contact the researcher directly. The researcher will take written consent before the interview commences; participants will have the chance to ask questions before the interview, and it will be stressed to the participant that they are under no obligation to take part and may withdraw from the study at any point during the interview, and that their participation has no influence on their medical care.

Healthcare professionals will be invited by email to participate in the study, with a follow-up email after 1 week; eligible participants will be identified by the clinical team, and the email will provide them with a participant information sheet. If a potential participant expresses interest, they will be asked to contact the researcher directly or asked permission for the researcher to contact them. An interview slot will be arranged at an appropriate time for the individual and written consent will be taken by the researcher.

\section{Data analysis}

Thematic analysis ${ }^{25}$ will be used to interrogate the interview data. Analysis will be conducted by two researchers, with experience in qualitative research, but no fixed views regarding the management or assessment of MBO. After an initial period of independent coding, meetings will be held between the two researchers to refine the themes and their concomitant categories. The analysis framework will be iteratively refined until all transcripts have been coded and a final thematic framework produced. ${ }^{25}$

Findings from phase I (SLR) and phase II (interviews) will be summarised in a report which addresses the main research question: what issues do patients, their companions and healthcare professionals consider to be most important in the management of symptoms of MBO, and what improvements would they consider meaningful? These data will also be synthesised with the qualitative data (phase-II interviews) identified through a rapid review of the qualitative literature. The report will seek to identify significant experiences and priorities among patients, their carers (if present) and healthcare professionals, that align with measurable outcomes, ${ }^{23}$ and can be taken forward into the initial outcomes list presented in phase III.

\section{Phase III: steering meeting and expert panel}

A steering meeting will be held to consolidate the findings of the SLR and interview study, to produce a list of outcomes to be taken forward into round 1 of the Delphi. The outcome list will be discussed and refined by an expert panel (consisting of approximately 10 academics/patient or carer research partners/healthcare professionals chosen for their recognised expertise in and/or experience of $\mathrm{MBO}$ ), following Conducting and REporting DElphi Studies (CREDES) in palliative care guidelines for the Delphi technique. ${ }^{26}$ 


\section{Phase IV: Delphi survey}

The Delphi survey will be carried out using Online Survey UK, an electronic bespoke survey format, which has previously been developed by the COMET Initiative for the use in COS development projects. A piloting round will be conducted with expert and lay members of the steering panel prior to wider stakeholder involvement. If issues needing resolution arise from this process, a second pilot round will be included. The survey will present the list of outcomes to stakeholders in two rounds, with a final consensus meeting to resolve points of difference. The aim of the process is to reach consensus, taking in a range of viewpoints: stakeholders will include patient representatives and healthcare professionals in the area of clinical oncology, colorectal surgery, gastroenterology, gynaecological surgery and specialist nursing. Anonymous results from the first round will be circulated between all stakeholder groups as a basis for the subsequent round of decision-making. Items on which the agreed threshold of consensus is reached in round 1 will be dropped from round 2. This technique is recognised by the COMET Initiative as appropriate to the development of $\mathrm{COS}^{23}{ }^{27}$

\section{Recruitment}

Identification of potential patient research partners will take place in tandem with recruitment for phase II of the study, via the same recruitment channels, and through formal carer or bereavement groups run by voluntary organisations. Healthcare professionals will be recruited from hospitals and hospices and by using the authors' professional networks. Participants will be recruited through telephone, email or post. No new participants will be invited following the completion of round 1 .

Based on the recruitment numbers in the previous studies and a pragmatic approach to reaching consensus, ${ }^{28} 29$ we will aim to recruit a minimum of 40 respondents to take part, including patients/companions, healthcare professionals and policy. Consent will be implicit through the participants' registration to take part and completion of the survey. Each participant will be represented in the survey documents by a unique identifier label; personal details will be kept confidential in a separate database on secure online storage.

\section{Delphi process}

The outcome list produced during phase III will be used to construct the Delphi survey. Panel members will be asked to score each outcome on a Likert scale ranging from 1 to $9^{27}$ (1-3-limited importance; 4-6-important and 7-9-critically important), relative to the other outcomes, in terms of the following question:

- Please consider each listed outcome and rate how important it is to measure a change in the outcome when providing palliative care to address the symptoms of $\mathrm{MBO}$ ?

In addition to scoring outcomes, free text boxes will allow comments to be gathered on each item in each round of the survey, to make decision-making visible and assist other participants to refine their decision-making in the following round. This will allow respondents to add additional outcomes in order to address any perceived gaps in the way that patients' experiences are measured.

We anticipate two rounds to refine and rank outcomes in order to reach consensus, followed by a consensus meeting to resolve any items on which agreement is not reached. For each outcome in each round, the distribution of scores will be summarised, and items scored as of limited importance by all respondents will be dropped from subsequent rounds; all other items will be carried forward. The preset agreement criterion for scoring outcomes at each stage will be $\geq 70 \%$, based on the previous studies describing the development of COS. ${ }^{30} 31$ Consensus will be reached if the majority of participants score a specific outcome similarly. Three categories will be assigned for each outcome:

- Consensus: in-70\% or more respondents within a stakeholder group rate the outcome as critically important and $15 \%$ or fewer rate the outcome as of limited importance.

- Consensus: out-70\% or more of participants rated an outcome as of limited importance and $15 \%$ or fewer rated the outcome as critically important.

- No consensus-none of the above criteria is met.

If $70 \%$ or more of the respondents agree that an outcome is of low importance in round 1 , this item will be dropped from round 2. If $70 \%$ or more of respondents agree that an outcome is of high importance in round 1 , this item will be dropped from round 2 and will be proposed for inclusion in the final COS. All other items will be carried forward. In round 2, participants will be shown the number of respondents and distribution of scores for each remaining outcome from all stakeholder groups, with their own score from round 1 . They will be asked to consider the responses from other Delphi participants and to rescore the items. If a participant changes their score from of limited importance $(<4$ in round 1$)$ to critically important in round 2 ( 7 or more) or from critically important in round 1 to of limited importance in round 2, they will be asked to provide the reason for this change. We will also examine any other changes in scores between rounds and summarise reasons given for these changes.

Items on which agreement was not reached will be discussed and resolved at the final consensus meeting. Outcomes from the Delphi survey classed as 'consensus in' across all groups will be proposed for inclusion in the final COS; outcomes classed as 'consensus out' will be proposed for exclusion.

\section{Consensus meeting}

All Delphi participants will be eligible for inclusion in a face-to-face consensus meeting to achieve a final recommendation of a core set of outcomes. The survey registration page for round 1 will ask participants to tick a box to allow the study team to approach them for inclusion. Appropriate secondary stakeholder groups recommended 
by existing stakeholders will be invited to take part at this stage. Written consent will be taken at the outset of the meeting from all participants to audio record the discussion; the audio file will be transcribed and anonymised. Participants will discuss all outcomes and their status at the close of the survey. They will then vote on an outcomeby-outcome basis to confirm consensus or to suggest that an outcome requires further discussion. Further voting rounds will be conducted as necessary until the final outcome set is agreed.

\section{ETHICAL CONSIDERATIONS}

Ethical approval for the study was obtained on 10 December 2019, from Wales Research Ethics Committee 5 Bangor (REF: 19/LO/1876). All data will be collected according to the principles of the General Data Protection Regulations and Data Protection Act 2018. ${ }^{32}$

\section{DISSEMINATION}

The study is registered with the COMET database and dissemination through this will complement peer-review publications. Study participants and relevant bodies (eg, healthcare professionals involved in the management of $\mathrm{MBO}$-including palliative care, gynaecology and colorectal surgery and patient partner organisations) will be regularly updated with newsletters and a lay summary at the end of the study. We will disseminate the research findings widely. Abstracts will be submitted to national and international conferences, result papers will be to relevant stakeholders at international conferences and submitted to publish in high-impact, open access journals. Dissemination will be undertaken with professional organisations such as the Association of Palliative Medicine, European Association for Palliative Care, The Multinational Association of Supportive Care in Cancer, The American Society of Clinical Oncology, The European Society for Medical Oncology, National Cancer Research Institute and Hospice UK to adopt the recommendations from the work. Lay summaries will be uploaded on the Marie Curie website, including the Marie Curie Palliative Care Research Centre (MCPCRC)-specific website and Wolfson Palliative Care Research Centre websites, and sent to key PPI groups and disseminated via social media.

\section{Author affiliations}

${ }^{1}$ Marie Curie Palliative Care Research Centre, Cardiff University School of Medicine, Cardiff, UK

${ }^{2}$ Wolfson Palliative Care Research Centre, Hull York Medical School, Hull, UK

${ }^{3}$ University of Technology Sydney, Sydney, New South Wales, Australia

${ }^{4}$ Queen's Centre for Oncology and Haematology, Hull, UK

${ }^{5}$ Dove House Hospice, Hull, UK

${ }^{6}$ Consumer Liaison Group, National Cancer Research Institute, London, UK

${ }^{7}$ Trans-Humber Consumer Research Panel, Hull, UK

\section{Twitter Annmarie Nelson @annmarie0 and Simon Noble @simonnoble}

Contributors JB, SN, MJ, DCC, FEMM, ElaB, GO, KS and AN contributed equally to the development of the study and writing of the protocol. EliB and $A B$ wrote the first draft of the manuscript. EliB, AB, JB, SN, MJ, DCC, FEMM, ElaB, GO, KS, A0 and AN contributed to the refinement of the manuscript with specific additional contributions as follows: clinical overview and research methodology (JB,SN, MJ, DCC, FEMM, ElaB and GO), systematic review (GO and JB), qualitative research (AN), PPI including interview schedules and participant information. JB and SN maintained oversight of the manuscript and are chief investigators of the study.

Funding This work is supported by the Marie Curie Research Grants Scheme, grant number MCRGS-20171220-8020.

Competing interests None declared.

Patient and public involvement Patients and/or the public were involved in the design, or conduct, or reporting, or dissemination plans of this research. Refer to the Methods section for further details.

Patient consent for publication Not required.

Provenance and peer review Not commissioned; peer reviewed for ethical and funding approval prior to submission.

Open access This is an open access article distributed in accordance with the Creative Commons Attribution Non Commercial (CC BY-NC 4.0) license, which permits others to distribute, remix, adapt, build upon this work non-commercially, and license their derivative works on different terms, provided the original work is properly cited, appropriate credit is given, any changes made indicated, and the use is non-commercial. See: http://creativecommons.org/licenses/by-nc/4.0/.

\section{ORCID iDs}

Elin Baddeley http://orcid.org/0000-0002-7571-4820

Jason Boland http://orcid.org/0000-0001-5272-3057

\section{REFERENCES}

1 Tuca A, Guell E, Martinez-Losada E, et al. Malignant bowel obstruction in advanced cancer patients: epidemiology, management, and factors influencing spontaneous resolution. Cancer Manag Res 2012;4:159-69.

2 Mercadante S. Intestinal dysfunction and obstruction. In: Walsh D, ed. Palliative medicine. Philadelphia, PA: Saunders/Elsevier, 2009: 1267-75.

3 Gemlo B, Rayner AA, Lewis B, et al. Home support of patients with end-stage malignant bowel obstruction using hydration and Venting gastrostomy. Am J Surg 1986;152:100-4

4 Currow DC, Quinn S, Agar M, et al. Double-Blind, placebo-controlled, randomized trial of octreotide in malignant bowel obstruction. J Pain Symptom Manage 2015;49:814-21.

5 Peng X, Wang P, Li S, et al. Randomized clinical trial comparing octreotide and scopolamine butylbromide in symptom control of patients with inoperable bowel obstruction due to advanced ovarian cancer. World J Surg Oncol 2015;13:50.

6 Kubota H, Taguchi K, Kobayashi D, et al. Clinical impact of palliative treatment using octreotide for inoperable malignant bowel obstruction caused by advanced urological cancer. Asian Pac J Cancer Prev 2013;14:7107-10.

7 Zhang Y, Gao Y, Ma Q, et al. Randomised clinical trial investigating the effects of combined administration of octreotide and methylglucamine diatrizoate in the older persons with adhesive small bowel obstruction. Dig Liver Dis 2006;38:188-94.

8 Khoo D, Hall E, Motson R, et al. Palliation of malignant intestinal obstruction using octreotide. Eur J Cancer 1994;30A:28-30.

9 Selby D, Wright F, Stilos K, et al. Room for improvement? A qualityof-life assessment in patients with malignant bowel obstruction. Palliat Med 2010;24:38-45.

10 Laval G, Marcelin-Benazech B, Guirimand F, et al. Recommendations for bowel obstruction with peritoneal carcinomatosis. J Pain Symptom Manage 2014;48:75-91.

11 NHS Scotland. Scottish palliative care guidelines: bowel obstruction Available: http://www.palliativecareguidelines.scot.nhs.uk/guidelines/ symptomcontrol/bowel-obstruction.aspx [Accessed 12 Dec 2019].

12 Mariani P, Blumberg J, Landau A, et al. Symptomatic treatment with lanreotide microparticles in inoperable bowel obstruction resulting from peritoneal carcinomatosis: a randomized, double-blind, placebo-controlled phase III study. J Clin Oncol 2012;30:4337-43.

13 Walsh D, Davis M, Ripamonti C, et al. 2016 updated MASCC/ESMO consensus recommendations: management of nausea and vomiting in advanced cancer. Support Care Cancer 2017;25:333-40.

14 Mercadante S. Octreotide for malignant bowel obstruction: commentary on Currow et al. J Pain Symptom Manage 2015;49:813.

15 Johnson MJ, Noble SIR. Challenging previously held beliefs about clinical practice: evidence or experience? J Pain Symptom Manage 2015;50:e1-2. 
16 Noble SIR, Murtagh FEM, Bausewein C, et al. Reply to: MASCC/ ESMO consensus recommendations for the management of nausea and vomiting in advanced cancer. Support Care Cancer 2017:25:2989-90.

17 Campbell R, McCaffrey N, Brown L, et al. Clinician-reported changes in octreotide prescribing for malignant bowel obstruction as a result of an adequately powered phase III study: a transnational, online survey. Palliat Med 2018;32:1363-8.

18 Obita GP, Boland EG, Currow DC, et al. Somatostatin analogues compared with placebo and other pharmacologic agents in the management of symptoms of inoperable malignant bowel obstruction: a systematic review. J Pain Symptom Manage 2016;52:901-19.

19 Health Research Authority, NHS. Best practice in research for outcome measures. Available: https://www.hra.nhs.uk/planningand-improving-research/best-practice/outcome-measures/ [Accessed 21 Jan 2020].

20 Heneghan C, Goldacre B, Mahtani KR. Why clinical trial outcomes fail to translate into benefits for patients. Trials 2017;18:122.

21 Saxby C, Ackroyd R, Callin S, et al. How should we measure emesis in palliative care? Palliat Med 2007;21:369-83.

22 Williamson PR, Altman DG, Bagley H, et al. The COMET Handbook: version 1.0. Trials 2017;18:280.

23 National Institute for Health Research. UK national standards for public involvement in research - PPI. Available: https://www.invo.org uk/current-work/standards/ [Accessed 21 Jan 2020].

24 NVIVO. NVivo12 qualitative software. Available: https://www. qsrinternational.com/nvivo/nvivo-products/nvivo-12-plus
25 Braun V, Clarke V. Using thematic analysis in psychology. Qual Res Psychol 2006;3:77-101.

26 Jünger S, Payne SA, Brine J, et al. Guidance on conducting and reporting Delphi studies (CREDES) in palliative care: recommendations based on a methodological systematic review. Palliat Med 2017;31:684-706.

27 Guyatt GH, Oxman AD, Kunz R, et al. GRADE guidelines: 2. Framing the question and deciding on important outcomes. J Clin Epidemiol 2011:64:395-400.

28 Firth AM, O'Brien SM, Guo P, et al. Establishing key criteria to define and compare models of specialist palliative care: a mixed-methods study using qualitative interviews and Delphi survey. Palliat Med 2019;33:1114-24.

29 Biondo PD, Nekolaichuk CL, Stiles C, et al. Applying the Delphi process to palliative care tool development: lessons learned. Support Care Cancer 2008:16:935-42.

30 Waters AM, Tudur Smith C, Young B, et al. The CONSENSUS study: protocol for a mixed methods study to establish which outcomes should be included in a core outcome set for oropharyngeal cancer. Trials 2014;15:168.

31 Fish R, Sanders C, Williamson PR, et al. Core outcome research measures in anal cancer (CORMAC): protocol for systematic review, qualitative interviews and Delphi survey to develop a core outcome set in anal cancer. BMJ Open 2017;7:e018726.

32 GDPR and data protection act 2018. Available: https://www.gov.uk/ government/collections/data-protection-act-2018 [Accessed 21 Jan 2020]. 